\title{
A Non-Invasive Experimental Approach for Surface Temperature Measurements on Semi-Crystalline Thermoplastics
}

\author{
Sinan Boztepe ${ }^{1}$, Remi Gilblas ${ }^{1}$, Olivier de Almeida ${ }^{1, a}$, Yannick Le Maoult ${ }^{1, \mathrm{~b}}$ and \\ Fabrice Schmidt ${ }^{1, \mathrm{c}}$ \\ ${ }^{1}$ Université de Toulouse ; Mines Albi, ICA (Institut Clément Ader) ; Campus Jarlard, F-81013 \\ Albi cedex 09, France. \\ a) olivier.dealmeida@mines-albi.fr, ${ }^{b}$ lemaoult@mines-albi.fr, ${ }^{c}$ fabrice.schmidt@mines-albi.fr
}

\begin{abstract}
Most of the thermoforming processes of thermoplastic polymers and their composites are performed adopting a combined heating and forming stages at which a precursor is heated prior to the forming. This step is done in order to improve formability by softening the thermoplastic polymer. Due to low thermal conductivity and semi-transparency of polymers, infrared (IR) heating is widely used for thermoforming of such materials. Predictive radiation heat transfer models for temperature distributions are therefore critical for optimizations of thermoforming process. One of the key challenges is to build a predictive model including the physical background of radiation heat transfer phenomenon in semi-crystalline thermoplastics as their microcrystalline structure introduces an optically heterogeneous medium. In addition, the accuracy of a predictive model is required to be validated experimentally where IR thermography is one of the suitable methods for such a validation as it provides a non-invasive, full-field surface temperature measurement. Although IR cameras provide a non-invasive measurement, a key issue for obtaining a reliable measurement depends on the optical characteristics of a heated material and the operating spectral band of IR camera. It is desired that the surface of a material to be measured has a spectral band where the material behaves opaque and an employed IR camera operates in the corresponding band. In this study, the optical characteristics of the PO-based polymer are discussed and, an experimental approach is proposed in order to measure the surface temperature of the PO-based polymer via IR thermography. The preliminary analyses showed that IR thermographic measurements may not be simply performed on PO-based polymers and require a correction method as their semi-transparent medium introduce a challenge to obtain reliable surface temperature measurements.
\end{abstract}

\section{INTRODUCTION}

Infrared (IR) radiation heat transfer is widely used for thermoforming process of thermoplastic polymers and their composites. For the case of heating unfilled thermoplastics, the key benefit of radiation heating is that a significant amount of the radiative energy penetrates directly into the bulk of polymer thanks to its semitransparency where high heat flux densities can be used to decrease the heating time. Thermoforming process typically involves heating and forming stages where thermoplastic precursors are heated prior to the forming in order to enhance the formability by virtue of softening the polymer. Therefore radiation heat transfer models are crucial for both temperature distributions on heated precursors and optimizations for the IR heating stage of thermoforming processes. Considering the thermoforming of unfilled semi-crystalline thermoplastics, the interaction between their microcrystalline structure and optical properties is the key to develop a successful predictive model as their microcrystalline structure introduces an optically heterogeneous medium. In addition, modeling radiation heat transfer in an optically heterogeneous medium is also required to be validated experimentally in order to quantify the accuracy of a predictive model. Temperature measurements alongside a heated material are commonly performed for the validation purposes where different measurement methods are employed. For surface temperature measurements on a heated material, IR thermography may be one of the suitable methods to measure the 
temperature accurately as it provides a non-invasive full-field measurement. Especially considering a radiation heating for polymers, conventional temperature measurement techniques such as contact thermocouples may cause to perturb heating process. It is known that contact thermocouples, which are made of metals, absorb radiation energy that is emitted from a radiative heating source and therefore causes to change the actual radiation energy absorbed by the surface of a heated material [1], [2]. This may change the actual temperature profile on the heated surface which eventually leads to have an erroneous measurement. Therefore, non-invasive temperature measurements are critical for the case of having measurement on radiatively heated surfaces.

For thermographic measurements using an IR camera it is required to know the optical characteristics of a heated material and the operating spectral band of an IR camera in order to obtain a reliable surface temperature measurement. In an ideal case, it is desired that the surface of a material to be measured has a spectral band where the material behaves opaque and an employed IR camera operates in the corresponding band. Opacity of a heated material determines how much absorbed radiation energy penetrates into the material medium, which is parametrized as optical penetration depth $\left(\mathrm{D}_{\mathrm{p}}\right)$ in radiation heat transfer theory [3], [4]. Due to semi-transparency of polymers extra attention may be given to analyze in which spectral band that the material is opaque [5] in order to adjust the operating spectral range of an employed IR camera for performing an accurate measurement. In addition, in terms of choosing a spectral band for thermographic surface measurement on semi-crystalline thermoplastics, the effect of microcrystalline structure may not be ignored as it may change the accuracy of measurement under heating. As the microcrystalline structure of such polymers may evolve under varying temperature it may cause to change the absorption characteristics and therefore $\mathrm{D}_{\mathrm{p}}$ of the polymer. Hence, it is critical to know in which spectral band that the optical characteristics of a semi-crystalline polymer are affected by its microcrystalline structure. Previous studies showed that the microcrystalline structure affects the optical characteristics of the PO-polymers in nearinfrared (NIR) range, whereas it may be negligible in middle-infrared (MIR) range [6], [7].

In this study, an experimental approach is proposed in order to measure the surface temperature of the PO-based polymer using IR thermography. Initially, the optical characteristics of the PO-based polymer are experimentally analyzed and discussed. Based on the optical characteristics of the polymer a spectral band is chosen where also an optical filter is employed regarding the chosen band and mounted on an IR camera. In addition, the optical penetration depth of the polymer is analyzed in the corresponding spectral band. For the preliminary IR thermographic analyses, the surface temperature of the polymer sample was registered using an IR camera and a resistance thermometer - also called resistance temperature detector (RTD)- and, the measurements obtained from both the methods are compared. The preliminary analyses showed that IR thermographic temperature measurements may not be simply performed on the PO-based polymers and require a correction method as their semi-transparent medium introduce a challenge to obtain reliable surface temperature measurements. In addition, such a correction method may only be developed adopting a comparative analysis based on the IR thermographs of the PO-based polymer and a reference surface that may be prepared using a reference material such as black coatings.

\section{EXPERIMENTAL PROCEDURE}

\section{Semi-transparency and optical characteristics of PO-based polymer}

In order to determine where the PO-based polymer shows no transmittance, its optical characteristics was analyzed in a previous study using a Fourier Transform Infrared Spectroscopy [7]. In Figure 1, the transmission characteristics of the polymer samples $(0.25,0.42,1.82 \mathrm{~mm}$ thick) are displayed in NIR (a) and MIR (b) ranges. As it seen in the figure, there are three narrow spectral bands where the PO-based polymer shows no transmittance which are around 3.4, 7.6 and $13.6 \mu \mathrm{m}$ respectively. Regarding whole the spectrum analyzed in NIR and MIR ranges, it can be stated that the material is highly transparent. It is known that carbon-hydrogen $(\mathrm{C}-\mathrm{H})$ absorption band around $3.4 \mu \mathrm{m}$ causes to have zero transmittance as reported for polypropylene in [8] or polyethylene in [9]. Whereas, our previous study demonstrated that the microcrystalline structure of the PO-based polymer greatly affects the transmission behavior for the wavelengths lower than $3.5 \mu \mathrm{m}$ where after this effect gradually decreases and becomes negligibly small for the wavelengths higher than $6 \mu \mathrm{m}$. A similar trend was also reported in [6] for polypropylene where it was concluded that the effect of microcrystalline morphology is negligible once an increase in the wavelength in MIR range is seen. This is critical in terms of performing an IR thermographic measurement under heating. Because the microcrystalline structure of a semi-crystalline thermoplastics may evolve under varying temperature and therefore change the optical characteristics that may eventually cause to change the accuracy of the measurements. Therefore, the most accurate surface temperature measurement may not be obtained using this spectral band. In addition, a typical IR camera has an operating spectral band between 7-14 $\mu \mathrm{m}$ [5] that covers the 
two other spectral bands that are around 7.6 and $13.6 \mu \mathrm{m}$. Hence, those spectral bands are the most suitable ones for IR thermographic measurements as the tested PO-polymer samples show no transmittance in the spectral range of a typical IR camera operates.

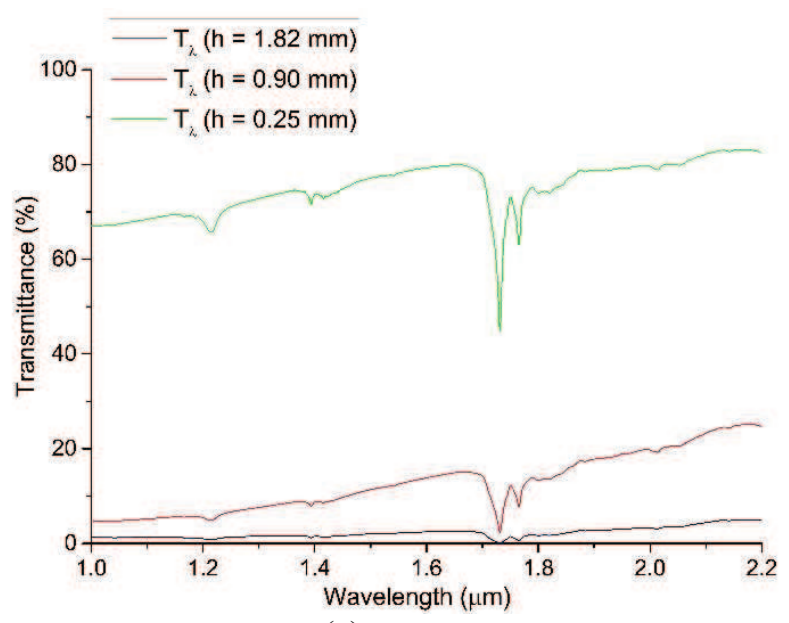

(a)

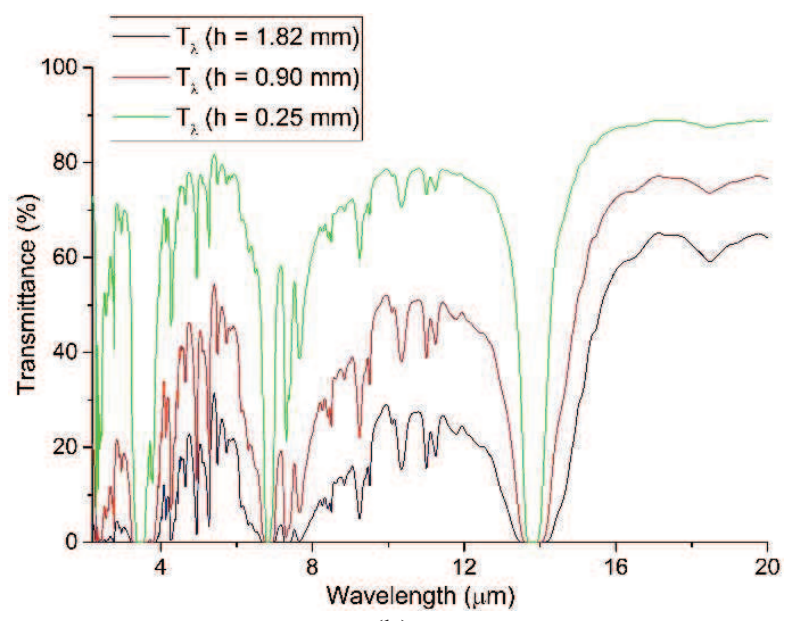

(b)

FIGURE 1. Transmission characteristics of the PO-based polymer samples in NIR (a) and MIR range (b).

The suitable spectral ranges are relatively narrow in comparison to an operating range of a typical IR camera. Therefore a tailored-optical filter is necessary for an accurate IR thermography. The term -tailored- is used here to define the specific bandwidth. In other words optical filters enable to adjust an IR camera to make it to capture photons only in preferably selected wavelengths however it also reduces the intensity of incident radiation received by the IR camera detector that may cause to have a low sensitivity. In the current study, at first step, two different optical filters were employed for the two suitable spectral ranges. The employed optical filters (Northumbria Optical Coatings Ltd.) have spectral ranges around $7.6 \mu \mathrm{m}$ and $13.6 \mu \mathrm{m}$, where their bandwidths are $\pm 0.10 \mu \mathrm{m}$ and $\pm 0.21 \mu \mathrm{m}$ respectively. The initial thermographic analyses showed that the sensitivity of the employed IR camera was too low around $13.6 \mu \mathrm{m}$. Therefore, the optical filter with a spectral range around $7.6 \mu \mathrm{m}$ was chosen for the IR thermographic measurements. For the case of IR thermography of PET polymer, no such filter is needed to have an accurate thermography on the polymer surface as PET can be assumed opaque in the whole operating range of a typical IR camera as studied in [5]. In addition, $\mathrm{D}_{\mathrm{p}}$ of PET is known to be around 30 micron at $400 \mathrm{~K}$ indicating that the total radiation energy emitted by the polymer is very close the surface [10]. For the PO-based polymer, the $\mathrm{D}_{\mathrm{p}}$ was calculated considering the chosen spectral band. As aforementioned, microcrystalline structure does not affect the optical characteristics of the PO-based polymer in the chosen spectral band, so homogeneous medium assumption can be adopted with $\mathrm{D}_{\lambda}=0$. Thus the transmittance $\left(\mathrm{T}_{\lambda}\right)$ - which is the ratio of the total amount of transmitted $\left(\Phi_{\lambda}{ }^{t}\right)$ radiative flux over the incident flux $\left(\Phi_{\lambda}{ }^{i}\right)$ - can be related only to the absorption characteristics where the extinction $\left(\beta_{\lambda}\right)$ and absorption $\left(\kappa_{\lambda}\right)$ coefficients become equal:

$$
T_{\lambda}=\frac{\phi_{\lambda}^{t}}{\phi_{\lambda}^{i}}=e^{-\beta_{\lambda} \cdot d} \text { with; } \beta_{\lambda}=\kappa_{\lambda}+\mathrm{D}_{\lambda} \text { where; } \quad \mathrm{D}_{\lambda}=0
$$

And the integrated absorption coefficient, which is reciprocal of $\mathrm{D}_{\mathrm{p}}$, can be calculated based on the transmittance of the PO-based polymer in the spectral band of the chosen optical filter $(7.5 \leq \lambda \leq 7.7 \mu \mathrm{m})$ and at the temperature $(\mathrm{T}=373 \mathrm{~K})$ close to the thermoforming process temperature of the polymer:

$$
\bar{\kappa}(T=373 K)=\frac{\int_{\lambda_{1}=7.5 \mu \mathrm{m}}^{\lambda_{2}=7.7 \mu \mathrm{m}} \kappa_{\lambda} \cdot I_{\lambda}^{o}\left(T_{373 K}\right) d \lambda}{\int_{\lambda_{1}=7.5 \mu \mathrm{m}}^{=7.7 \mu \mathrm{m}} I_{\lambda}^{o}\left(T_{373 K}\right) d \lambda} \quad \text { with; } \mathrm{D}_{\mathrm{p}}=\frac{1}{\bar{\kappa}(T=373 K)}
$$


In the corresponding spectral band, the $\mathrm{D}_{\mathrm{p}}$ of the polymer was found around $350 \mu \mathrm{m}$ indicating that the absorbed and therefore emitted radiation may not be considered on the surface of the polymer but in a range close to surface which may induce a correction on the thermographic measurement. Therefore the energy emitted by the heated polymer surface may be needed to be corrected in order to have an accurate temperature measurement.

\section{IR camera Calibration and Test-up for Thermographic Analyses}

For the IR thermographic measurements a cooled long-wave (LW) IR camera (AGEMA 880) was employed and the chosen narrow-band optical filter was mounted on the IR camera. Before doing IR thermographic analyses with the employed LW IR camera, the optical filter was also mounted on an uncooled microbolometric IR camera (FLIR SC325) and tested however enough sensitivity could not be obtained. The typical operating spectral range of the employed LW IR camera is between 8 and $12 \mu \mathrm{m}$ indicating that spectral sensitivity of the IR camera detector is nominal in this range, whereas the detector is still sensitive on the wavelengths beyond, but close to, this range. In the current study, it was found out that the LW IR camera is still sensitive enough for the measurements in the spectral band around $7.6 \mu \mathrm{m}$, however an extra step was required to be applied to adjust the sensitivity of the camera. In order to establish a correlation between the IR radiation captured by the camera and a surface temperature to be measured a blackbody calibration source (Landcal P550P) was used. The calibration was done choosing reference temperatures on the blackbody source between 60 and $120^{\circ} \mathrm{C}$ and the radiometric value (UI) detected by the camera was registered for each reference temperature. Thanks to this step, a calibration curve was established that is required to convert the energy to be emitted from the surface of the PO-based polymer (and detected by the IR camera) to a temperature value. In figure 2, test set-up for the calibration (a) and the registered radiometric values in the chosen reference temperatures (b) are displayed.

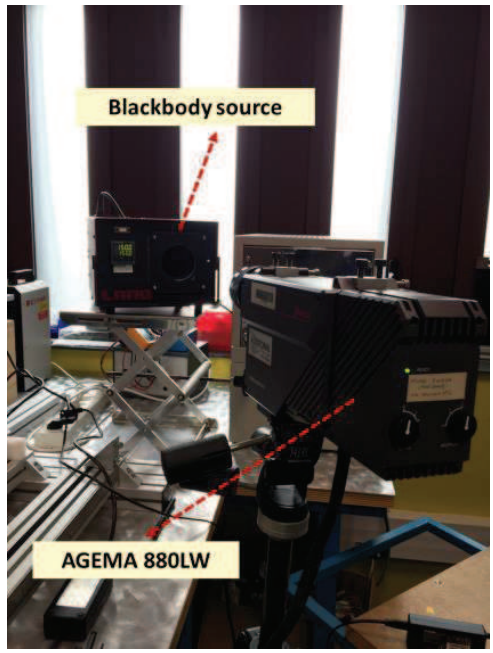

(a)

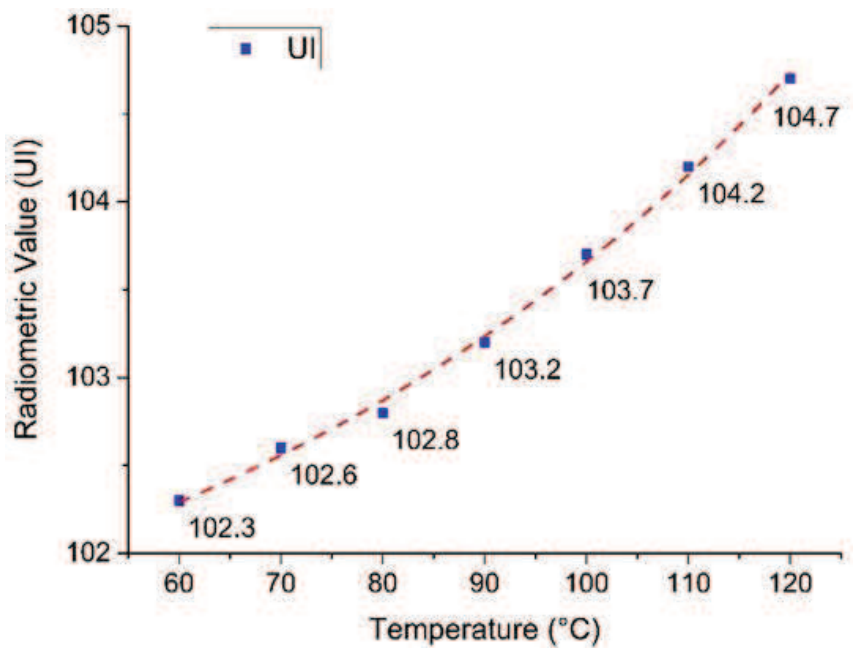

(b)

FIGURE 2. Test set-up for calibration of IR camera with the optical filter at $7.6 \mu \mathrm{m}$ (a) and the calibration curve obtained for the reference temperatures between 60 and $120{ }^{\circ} \mathrm{C}(\mathrm{b})$.

Thanks to the calibration curve, a preliminary IR thermographic analysis was done on the PO-based polymer. For the analysis, the polymer was fabricated in the form of rectangular sheet and the sample was heated by conduction using an electric thermofoil heater (Minco). In order to perform a homogeneous heat transfer from the thermofoil heater throughout sample geometry a copper foil was embedded between the heater and the sample. In addition, black coating was applied on the two small zones of the sample surface for creating reference measurement zones, where its emissivity was previously measured as 0.9 in our laboratory. It should be mentioned at this point that, for the preliminary analyses, the contact between all the components was secured using a clamp on each side whereas the thermal contact may be improved in the next steps of the analysis using thermal grease at the interfaces of the components. The test set-up for heating the PO-based sample is displayed in Figure 3. 


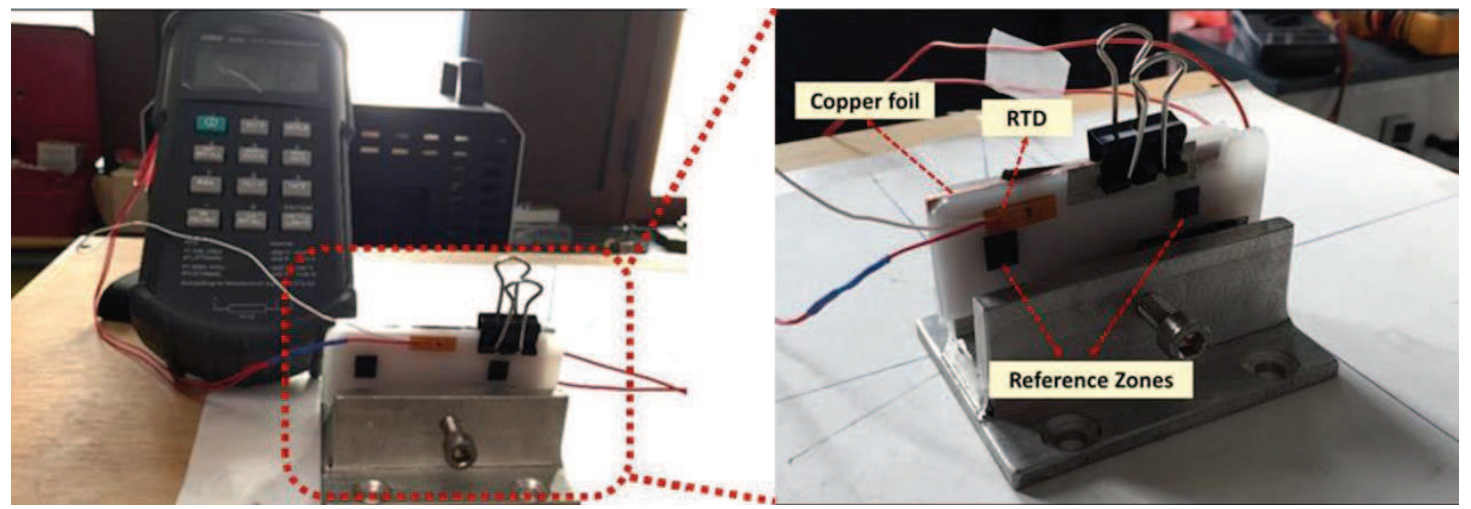

FIGURE 3. Test set-up for heating the PO-based polymer with a resistance thermometer for surface temperature measurement

\section{RESULTS AND DISCUSSION}

\section{IR Thermography of PO-Based Polymer}

The surface temperature measurements were performed between 60 and $80^{\circ} \mathrm{C}$ where the measurements were registered at each $5^{\circ} \mathrm{C}$ increment via both the RTD and the IR camera. So five measurements were obtained in the chosen temperature range and the energy detected by the IR camera during the heating of the polymer sheet was converted into temperature thanks to the obtained calibration curve. The registered IR thermographs of the PO-based polymer were compared to the RTD measurements obtained at the corresponding incremental measurement step. In Figure 4, a thermographic measurement obtained during the heating of the polymer sheet (a) and the recorded temperature values that were obtained via the RTD and the IR camera at each measurement step (b) are presented. As displayed in Figure 4(a), a measurement zone was defined and the same zone was analyzed on each IR thermographic image obtained in each measurement step to make comparison to the RTD measurements. The measurement zone was chosen close to the area where the RTD was positioned on the sample surface. The comparisons between the surface temperature measurements showed that error in the recorded values is less than $1 \%$ for the measurements obtained at 60 and $65^{\circ} \mathrm{C}$ whereafter the temperature read by the IR camera shows higher value than the RTD measurements at higher temperatures and the error reaches to $17.5 \%$ at which the difference in the measured temperatures is $15^{\circ} \mathrm{C}$. The reason behind this may be twofold: The temperature measurement obtained via RTD may be erroneous due to the polymer softening at higher temperatures as it may change the contact between the RTD and the polymer surface. Also, a potential thermal resistance alongside the metallic wires of the RTD may affect the measured temperature at which the heat losses increase proportionally with an increase in temperature. An accurate comparison may be obtained between the IR thermography and the reference measurement zone created using black coating.

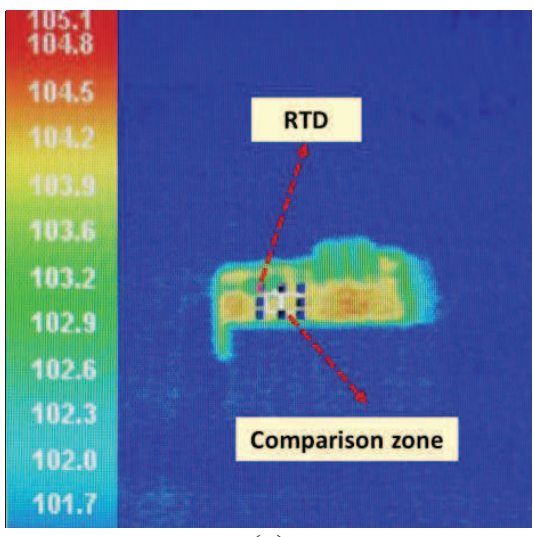

(a)

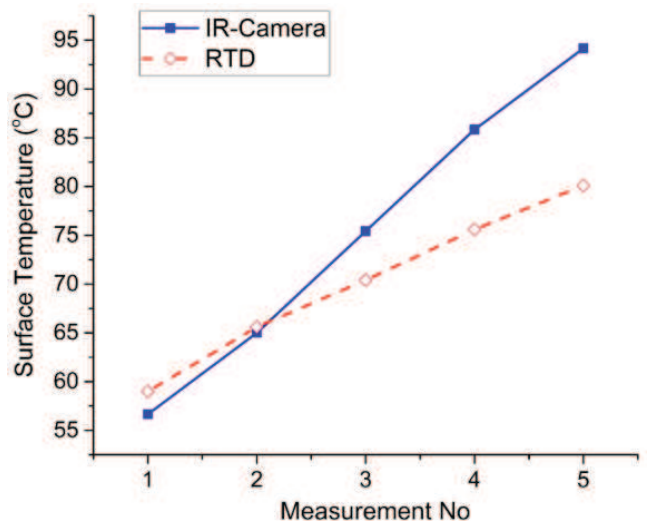

(b)

FIGURE 4. IR thermography of the PO-baser polymer at $75 \mathrm{C}$ (a) and the comparison between the measured surface temperatures obtained through the resistance thermometer and the IR camera (b) 


\section{CONCLUSIONS}

In order to validate radiation heat transfer modeling temperature measurements alongside a heated material is commonly performed where different measurement methods are employed. For radiation heating of polymers, IR thermography may be one of the suitable methods to measure the temperature accurately as it provides non-invasive full-field surface measurement on a heated zone. The conventional measurement techniques such as contact thermocouples may cause to perturb a radiation heating process and lead to have an erroneous measurement.

For performing an accurate IR thermographic measurement, it is ideal that a material behave opaque in the operating spectral range of an IR camera where its optical penetration depth is very low and therefore all the absorbed radiation energy can be assumed on the heated surface. The analysis on the optical properties of the PObased polymer showed that the material has several narrow spectral bands where it has zero transmittance for the samples with a thickness of the polymer samples with the thicknesses of $0.25,0.42,1.82 \mathrm{~mm}$ respectively. Based on the transmittance measurements, the optical penetration depth of the PO-based polymer was found around $350 \mu \mathrm{m}$ indicating that all the absorbed radiation energy by the polymer may not be considered on its surface but in a range. These analyses provided a guide to develop an experimental approach for the IR thermography of the PO-based polymer. A preliminary IR thermographic analysis of the polymer was performed and its surface temperature was also registered using a RTD at which those measurements were compared to the ones obtained through the IR camera. The preliminary analyses demonstrated that IR thermographic measurements can be obtained on the semitransparent PO-based polymer using a tailored optical filter. However a precise measurement may only be obtained after a correction methodology is applied as the optical penetration depth of the polymer introduces a challenging step in terms of emitted energy by its surface. These preliminary results clearly showed that the correction method may be developed adopting a comparative analysis between IR thermographs of polymer and the reference measurement zone created using a black coating while the measurement obtained via RTD may not be used as a reference temperature due to challenges in measurement accuracy during heating of the polymer.

\section{REFERENCES}

1. J. Jones, S. P. Brookes, M. T. Whittaker, and R. J. Lancaster, “, Mater. Sci. Technol. 30, 1862-1876 (2014).

2. Y. Luo, L. Chevalier, F. Utheza, and X. Nicolas, "Simplified Modelling of the Infrared Heating Involving the Air Convection Effect before the Injection Stretch Blowing Moulding of PET Preform," in $17^{\text {th }}$ ESAFORM Conference - 2014, 611, pp. 844-851.

3. J. R. Howell, M. P. Menguc, and R. Siegel, Thermal Radiation Heat Transfer, 5th Edition (CRC Press, Boca Raton, 2010).

4. R. Wester, Ed., Energy Transport and Heat Conduction in Tailored Light 2, edited by R. Poprawe, (Springer, Berlin Heidelberg, 2011), pp. 43-62.

5. Y. Le Maoult and F. Schmidt, "Infrared Radiation Applied to Polymer Processes," in Heat Transfer in Polymer Composite Materials, edited by N. Boyard, (John Wiley \& Sons, Inc., 2016), pp. 385-423.

6. D. Hakoume, L. A. Dombrovsky, D. Delaunay, and B. Rousseau, "Effect of Processing Temperature on Radiative Properties of Polypropylene and Heat Transfer in the Pure and Glassfibre Reinforced Polymer," in $15^{\text {th }}$ International Heat Transfer Conference- 2014.

7. S. Boztepe, A. Thiam, O. de Almeida, Y. Le Maoult, and F. Schmidt, "Experimental analysis on the coupled effect between thermo-optical properties and microstructure of semi-crystalline thermoplastics," in 19th ESAFORM Conference - 2016, AIP Conference proceedings, pp. 020006.

8. A. Bendada, K. Cole, M. Lamontagne, and Y. Simard, J. Opt. Pure Appl. Opt. 5, 464-470 (2003).

9. D. P. DeWitt and G. D. Nutter, Theory and Practice of Radiation Thermometry. (John Wiley \& Sons, Inc., 1988).

10. S. Monteix, Y. L. Maoult, F. Schmidt, and J. P. Arcens, Quant. InfraRed Thermogr. J. 1, pp. 133-150 (2004). 